\title{
Adaptive Actuator Compensation of Position Tracking for High-Speed Trains with Disturbances
}

\author{
Zehui Mao, Gang Tao, Fellow, IEEE, Bin Jiang, Senior Member, IEEE, Xing-Gang Yan
}

\begin{abstract}
In this paper, the adaptive fault compensation problem is investigated for high-speed trains in the presence of timevarying system parameters, disturbances and actuator failures. To deal with the time-varying system parameters, a new timevarying indicator function instead of commonly used 0-1 function, is proposed to model the train dynamics as a piecewise model with unparameterizable time-varying disturbances, which can cover more time variations and help parametrization for adaptation. A backstepping adaptive controller is designed for the healthy system with unknown piecewise model parameters and known piecewise bounds on disturbances. For both the parameterizable and unparameterizable failures, the backstepping adaptive failure compensation with the adaptive laws are derived to achieve the position tracking under the known bound disturbances. The adaptive failure compensation for unknown bounds on disturbances is also discussed under the parameterizable failure. Through introducing the nonlinear damping in the proposed controller, the failure compensation controller is proposed for the model with unparameterizable system parameters to achieve an arbitrary degree of position tracking accuracy. The stability of the corresponding closed-loop system and asymptotic state tracking are proved via Lyapunov direct method, and validated using a high-speed train model.
\end{abstract}

Index Terms-Failure compensation, actuator failures, adaptive control, high-speed train.

\section{INTRODUCTION}

High-speed train with its reliable, fast and high loading capacities, has attracted more and more attention in the recent years. With the increasing requirements of the speed and safety of the train operation, a lot of efforts has been devoted to the control design for high-speed trains, see [1]- [3]. Similar to the other complex systems, high-speed train formed by the sensors, motors, electrical components, mechanical drives and so on, will have failures with the long time (distance) operation, which could lead to the delay and even stop of the train. Thus, it is critical to study fault diagnosis and fault-

Copyright (c) 2015 IEEE. Personal use of this material is permitted. However, permission to use this material for any other purposes must be obtained from the IEEE by sending a request to pubs-permissions@ieee.org.

This work was supported in part by the National Natural Science Foundation of China under Grant 61490703 , Grant 61573180 and in part by the USA NSF under Grant ECCS1509704. (Corresponding author: Bin Jiang.)

Z. Mao and B. Jiang are with College of Automation Engineering, Nanjing University of Aeronautics and Astronautics, Nanjing, 210016, China, Email: zehuimao@nuaa.edwcn (Z. Mao), binjiang@nuaa.edu.cn (B. Jiang, corresponding author)

G. Tao is with Deparment of Electrical and Computer Engineering, University of Virginia, Charlottesville, VA 22904, U.S.A, and he is a visiting professor in Nanjing University of Aeronautics and Astronautics, Email: gt9s@virginia.edu.

X. Yan is with School of Engineering and Digital Arts, University of Kent, Canterbury, Kent CT2 7NT, United Kingdom, Email: x.yan@kent.ac.uk. tolerant control (failure compensation) problem for high-speed trains.

Although in the past years, a considerable amount of research have been made in fault diagnosis and fault-tolerant control (see, for example, [4]- [7]), the corresponding results for high-speed trains are very few (see, [8]- [9]). The existing results of the fault-tolerant control or the controller design show that the model-based methods are popular for the automatic train operation of high-speed trains. It is well-known that the dynamic motion model of the train is time-varying and nonlinear, changing with the train operating conditions. To deal with the characteristics of time-varying and nonlinearity, the time-invariant nonlinear model with bounded (Lipschitz) functions of disturbances is considered in [10] and [11]. However, disturbances under different operating conditions may not be the same, for example, the disturbances caused by the tunnel and the wheel-rail skid. It should be noted that one common bounded function on all disturbances cannot represent the characteristics of train dynamics well, which motivates us to utilize the piecewise disturbances to model the train dynamics. Further, considering that the change of the parameters of the train dynamic motion model are not abrupt, a piecewise model with time-varying indicator functions and bounded piecewise disturbances are considered in this paper, which is more practical and important.

Although there exist a lot of methods to obtain the system parameters (see, [12]- [14] ), it is difficult to obtain all the parameters in the train dynamic model, and the time and amplitudes of the failures. Moreover, position and speed tracking is one of the main tasks for the automatic train operation, which is implemented by the designed controller or fault-tolerant controller. The adaptive technique is proposed to solve the unknown parameters problem and to achieve good tracking performance (see [15], [16], [17]). To the best of the authors' knowledge, the failure compensation problem for piecewise model involved in the unknown parameters, failures and disturbances, has not been fully investigated yet.

This paper is focused on the actuator failure compensation problem for high-speed trains in the presence of parameterized time-varying parameters, unparameterized disturbances, and time-varying yet unpredictable actuator failures, simultaneously. The main contribution of this paper can be summarized as follows:

(i) Considering the time-varying system parameters and unmodelled disturbances of high-speed trains, a new time-varying indicator function which can represent more time variations than that $0-1$ indicator function represents, is proposed to parameterize the time-varying 
system parameters, and piecewise bounds of disturbance are introduced to describe the unparameterizable disturbances.

(ii) For different failure and disturbance cases (including the parameterized and unparameterized actuator failures with unknown failure time and unknown system parameters, the unknown bounds of disturbance), the adaptive failure compensation controllers with structure and adaptive laws are developed to achieve position tracking.

(iii) For the unparameterizable system parameters, the nonlinear damping is introduced into the adaptive failure compensation controller to achieve an arbitrary degree of tracking accuracy.

The rest of this paper is organized as follows: In Section II, the piecewise model with time-varying indicator functions and piecewise disturbances of high-speed trains is briefly introduced, and the actuator failure compensation issue is formulated. In Section III, an adaptive controller is designed for the healthy system with known disturbance bounds. In Sections IV and V, the failure compensation controllers for parameterized and unparameterized failures, are developed, respectively. In Section VI, under time-varying faulty condition, an adaptive compensation controller is presented for the unknown disturbance bounds. In Section VII, an adaptive compensation controller with nonlinear damping is proposed for the unparameterizable system parameters. In Section VIII, several simulation results for various operation conditions are presented to verify the effectiveness of the presented failure compensation controllers. Finally, conclusions are given in Section IX.

\section{Problem Formulation}

Considered the unmodelled uncertainties and the changes of the model parameters induced by the change of track conditions (e.g., slope, curve, tunnel, etc.), the piecewise model with time-varying indicator functions and piecewise disturbances is constructed in this section.

\section{A. Longitudinal Motion Dynamic Model}

The general dynamical model of longitudinal motion of the high-speed train is described as [18]

$$
\begin{aligned}
M(t) \ddot{x}(t)= & F(t)-a_{r}(t)-b_{r}(t) v(t)-c_{r}(t) v^{2}(t) \\
& -F_{g}(t)-F_{c}(t)+d(t),
\end{aligned}
$$

where $x(t)$ is the displacement of the train, $M(t)$ is the mass of the train, $F(t)$ is the traction force, $a_{r}(t)$ defines the train's rolling resistance component, $b_{r}(t)$ defines the train's linear resistance, $c_{r}(t)$ defines the train's nonlinear resistance; $v(t)$ is the speed of the train, $F_{g}(t)$ is the force caused by motion on the grade, $F_{c}(t)$ is the force caused by motion on the curve, $d(t)$ is the unmodelled factor, and can be the wheel-rail adhesion from rail or the nonlinear environmental disturbances from weather conditions. The traction force $F(t)$ is generated by the traction system in the train.
The model to describe the grade resistance force $F_{g}(t)$ and the curvature force $F_{c}(t)$ is given by (see [19]):

$$
\begin{aligned}
& F_{g}(t)=M(t) g \sin \theta(t), \\
& F_{c}(t)=0.004 D(t) M(t),
\end{aligned}
$$

where $\theta(t)$ is the slope angle of the current track, $D(t)$ is the degree of curvature.

Remark 1: There exist several models to express the curvature force $F_{c}(t)$, see [18]. Although the formulae are different, the expressions of the curvature force are in the completely parameterized form with the parameters related to the train wheelsets and curve radius. Here, we use the curvature force model from [19] to study the failure compensation problem. The proposed method can be employed to the other formulae of the curvature force.

Further, substituting expressions (2)-(3) into equation (1) yields

$$
\begin{aligned}
M(t) \ddot{x}(t)= & F(t)-\left(a_{r}(t)+b_{r}(t) \dot{x}(t)+c_{r}(t) \dot{x}^{2}(t)\right) \\
& -M(t) g \sin \theta(t)-0.004 D(t) M(t)+d(t) .
\end{aligned}
$$

As the displacement and velocity of a train can be measured online by the speed sensors and track circuits, the change time instants of these parameters can be obtained on-line.

Coefficients analysis. The mass is static when a train operated on line, which implies that the variable mass $M(t)$ can be modeled as a piecewise constant function depending on the displacement $x$ of the train.

According to the modelling methods in [18], it can be concluded that changes of coefficients $a_{r}, b_{r}$ and $c_{r}$ in (1) mainly depend on the current operation condition of the train (including mass, speed, tunnel passing, etc.). These coefficients $a_{r}, b_{r}, c_{r}, \theta(t)$ and $D(t)$ can be considered as constants in usual, when the whole train is running under a certain operation condition.

However, there are also some cases that parameters are varying even under some certain operation conditions. For example, due to the train length, the grade resistance force will increases to a maximum value from zero and decreases to zero, when the train is climbing a slope. The value of parameter $c_{r}(t)$ under the case that train just enters the tunnel, is different from that when the whole train is running in the tunnel, if the tunnel is long enough. For these rare typical situations, a piecewise constant with a known time-varying function can be introduced to improve the modelling accuracy.

Disturbance analysis. The disturbance $d(t)$ represents the unmodelled factors caused by changes of train operation conditions, such as rail, wheel, weather, etc. When the train is running under a certain operation condition, an upper bound can be used for the disturbance. With changes of the train operation condition, another upper bound should replace the former one.

Using the existing modelling methods, the upper bound of the disturbance $d(t)$ can be obtained. When the train operates on the horizontal line without slope or curvature under normal environment, the upper bound of the disturbance term $d(t)$, can be chosen as zero or a small positive scalar. When there is strong wind, the disturbance term $d(t)$ should be changed 
with the wind. According to the wind strength that the train can tolerate, the upper bound of $d(t)$ related to the strong wind can be estimated from the experiment data. The wheelrail adhesion is formulated as a nonlinear function with timevarying parameters, which is dependent on the wheel skid condition. The regions of the time-varying parameters can be obtained from the existing results about the wheel-rail adhesion (see [20]). Thus, the upper bound of $d(t)$ contributed to the wheel-rail adhesion can be calculated.

On the other hand, the train runs based on the operation timetable, and always moves on the same track at a given time interval and spatial interval, which makes the operation feature of train dynamic model in repeatable pattern [21], [22]. Based on the historical operating data, the bounds of the disturbances can be estimated.

From now on, it is concluded that the upper bound of the disturbance term $d(t)$ can be modelled as piecewise with known values. Due to the available information of the train (speed, weather, rail, ect.,), the change time instants of the train operation condition is available in real time. Similarly, the change time of disturbance bound can be obtained in real time as well.

Remark 2: Although common known bound can be employed to the disturbance, the piecewise model for disturbances is more accurate. Here, the piecewise constants are assumed known, which can be obtained from the physical experiments or practical measure. In the case when the piecewise constants are unknown, modified adaptive laws can be proposed, which will be considered later in this paper. Moreover, there are other types of disturbances, such as that discussed in [23] and [24], which could be considered in the future work.

\section{B. Piecewise Dynamic Model}

From the above analysis, it concludes that the parameters $M(t), a_{r}(t), b_{r}(t), c_{r}(t), \theta(t), D(t)$ and the bound on $d(t)$ are unknown piecewise constants or time-dependent functions, and are dependent on the velocity $\dot{x}$ and position $x$ of the train.

$$
\text { Let } m(t)=\frac{1}{M(t)}, a(t)=\frac{a_{r}(t)}{M(t)}, b(t)=\frac{b_{r}(t)}{M(t)}, c(t)=
$$

$\frac{c_{r}(t)}{M(t)}, \vartheta(t)=\sin \theta(t)$, and $\bar{d}(t)=\frac{d(t)}{M(t)}$. Equation (4) can be rewritten as

$$
\begin{aligned}
\ddot{x}(t)= & m(t) F(t)-\left(a(t)+b(t) \dot{x}(t)+c(t) \dot{x}^{2}(t)\right) \\
& -g \vartheta(t)-0.004 D(t)+\bar{d}(t),
\end{aligned}
$$

where $m(t), a(t), b(t), c(t), \vartheta(t), D(t)$ and the bound on $\bar{d}(t)$ are unknown piecewise constants or time-dependent functions.

To describe the parameters in equation (5) via a formal mathematical expression, the indicator functions $\chi_{\varrho i}(t)$ are introduced as:

$$
\chi_{\varrho i}(t)= \begin{cases}\Psi_{\varrho i}(t), & \text { if }(x(t), \dot{x}(t)) \in \Omega_{i}, \\ 0, & \text { otherwise, }\end{cases}
$$

where $\varrho \in\{m, a, b, c, \vartheta, D, \bar{d}\}, \Psi_{\varrho i}(t)$ is a known timedepending function satisfying $\Psi_{\varrho i}(t) \in(0,1]$. $\Psi_{\varrho i}(t)$ could be a constant 1 or a time-varying function. When $\Psi_{\varrho i}(t)$ is chosen as the constant 1 , the indicator function $\chi_{\varrho i}(t)$ is the common used 0-1 function, which would represent a piecewise constant model, as often used in the literature. When $\Psi_{\varrho i}(t)$ is a timevarying function, it could represent in ramp or exponential function form, which should increase from 0 to 1 and keep in 1 , or decrease from 1 to 0 and keep in 0 . Considering that the train mass can only change at the stations, the indicator function $\chi_{m i}(t)$ must be a $0-1$ function.

During train operation, the region for all possible system states $(x(t), \dot{x}(t))$ is denoted by $\Omega$ and its $l$ subregions are denoted by $\Omega_{i}, i=1, \ldots, l$. Further, when $(x(t), \dot{x}(t)) \in \Omega_{i},(m(t), a(t), b(t), c(t), \vartheta(t), D(t), \bar{d}(t))=$ $\left(m_{i} \chi_{m i}(t), a_{i} \chi_{a i}(t), b_{i} \chi_{b i}(t), c_{i} \chi_{c i}(t), \vartheta_{i} \chi_{\vartheta i}(t), D_{i} \chi_{D i}(t)\right.$, $\left.\bar{d}_{i}(t) \chi_{\bar{d} i}(t)\right), i=1, \ldots, l$, where $m_{i}, a_{i}, b_{i}, c_{i}, \vartheta_{i}$, and $D_{i}$ are unknown constants, $\left|\bar{d}_{i}(t)\right| \leq d_{i}^{0}$ and $d_{i}^{0}$ is known constants. Because $x(t)$ and $\dot{x}(t)$ are available in real-time, the time instants that $(x(t), \dot{x}(t))$ jumps from one region $\Omega_{i}$ to another $\Omega_{j}(i \neq j)$, are known. So, the functions $\chi_{\varrho i}(t)$ defined in (6) are known.

It is assumed that $(x(t), \dot{x}(t))$ only belongs to one region. Let $x_{1}=x$ and $x_{2}=\dot{x}$. The longitudinal motion dynamics (4) can be described by:

$$
\begin{aligned}
\dot{x}_{1}(t)= & x_{2}(t), \\
\dot{x}_{2}(t)= & m(t) F(t)-a(t)-b(t) x_{2}(t)-c(t) x_{2}^{2}(t) \\
& -g \vartheta(t)-0.004 D(t)+\bar{d}(t),
\end{aligned}
$$

where

$$
\begin{aligned}
m(t) & =\sum_{i=1}^{l} m_{i} \chi_{m i}(t), \quad a(t)=\sum_{i=1}^{l} a_{i} \chi_{a i}(t), \\
b(t) & =\sum_{i=1}^{l} b_{i} \chi_{b i}(t), \quad c(t)=\sum_{i=1}^{l} c_{i} \chi_{c i}(t), \\
\vartheta(t) & =\sum_{i=1}^{l} \vartheta_{i} \chi_{\vartheta i}(t), \quad D(t)=\sum_{i=1}^{l} D_{i} \chi_{D i}(t), \\
\bar{d}(t) & =\sum_{i=1}^{l} \bar{d}_{i}(t) \chi_{\bar{d} i}(t)
\end{aligned}
$$

with $m_{i}, a_{i}, b_{i}, c_{i}, \vartheta_{i}$, and $D_{i}$ being unknown constants; $\left|\bar{d}_{i}(t)\right| \leq d_{i}^{0}$ with $d_{i}^{0}$ being known constants, and $\chi_{\varrho i}(t)$ being the indicator functions defined in (6). Because bounds on $d(t)$ is known, and the masses of the empty and full train are also known, the bounds of the term $\bar{d}(t)=d(t) / M(t)$ is available.

Remark 3: Here, the known time-varying functions $\Psi_{\varrho i}(t)$ are introduced to improve the modelling accuracy, although the 0-1 indicator function can represent the most situations. There are only some typical situations, for which we choose some more accurate indicator functions. Thanks to the repeated train operating pattern, the functions $\Psi_{\varrho i}(t)$ can be obtained through training the historical data or analyzing the train motion. On the other hand, if the time-varying functions $\Psi_{\varrho i}(t)$ cannot be obtained, the model with unparameterizable system parameter is used to describe the high-speed train, whose controller designed will be presented later in this paper. 


\section{Actuator Failure Model}

The actuator failures in traction system are always generated by the failed equipments, which are modelled as constants or time-dependent functions. Generally, two types of models are usually used to describe the failure, i.e., parametric and nonparametric expressions. In this subsection, these two types of failure models will be introduced, and the adaptive failure compensation controller design for each type of failure models will be proposed.

Parameterized failure model. Consider $n$ motors in a highspeed train. The completely parametric failure model can be expressed as (see, e.g. [17])

$$
\begin{gathered}
F_{j}(t)=\bar{F}_{j}(t)=\bar{F}_{j 0}+\sum_{\rho=1}^{s_{j}} \bar{F}_{j \rho} f_{j \rho}(t), \\
t \geq t_{j}, \quad j \in\{1,2, \ldots, n\},
\end{gathered}
$$

where $j$ is the failure index, $t_{j}$ is the failure occurring time instant, $\bar{F}_{j 0}$ and $\bar{F}_{j \rho}$ are unknown constants. The basis signals $f_{j \rho}(t)$ are known, with $s_{j}$ being the number of the basis signals of the $j$ th actuator failure.

This failure model (11) covers several practical failure conditions of the high-speed train actuators, which is shown as follows:

1) Total failure. The motor stopping failure is a total failure. Then, (11) can be written as $F_{j}(t)=\bar{F}_{j}(t)=\bar{F}_{j 0}=0$, with $\bar{F}_{j \rho}=0$, for $\rho=1, \ldots, s_{j}$.

2) Constant failure. The mechanical drives locked failure can lead to the constant torque, which results in a constant actuator failure. Then, (11) can be written as $F_{j}(t)=\bar{F}_{j}(t)=$ $\bar{F}_{j 0}=$ non-zero constant, with $\bar{F}_{j \rho}=0$, for $\rho=1, \ldots, s_{j}$.

3) Periodic form failure. The IGBT (Insulated Gate Bipolar Transistor) failure (from PWM) can lead to the periodic failure with approximate known frequency, which could be a sine function. Then, (11) can be written as $F_{j}(t)=\bar{F}_{j}(t)=$ $\bar{F}_{j 1} \sin (w t)$ for some known $w$, with $\bar{F}_{j 0}=0, \bar{F}_{j 1}=$ non-zero unknown constant and $\bar{F}_{j \rho}=0$, for $\rho=2, \ldots, s_{j}$.

Since there are $n$ motors in the high-speed train, the resultant traction force $F(t)$ is the sum of the forces $F_{j}$, $j=1, \ldots, n$, generated from the $j$ th motor, given by:

$$
F(t)=\sum_{j=1}^{n} F_{j}(t) .
$$

From (11) and (12), the input of system (7)-(8) can be rewritten as

$$
\begin{aligned}
F(t) & =k_{\nu} \nu_{0}(t)+\xi^{T} \varpi(t), \\
\xi & =\left[\xi_{1}^{T}, \xi_{2}^{T}, \ldots, \xi_{n}^{T}\right]^{T}, \\
\xi_{j} & =\left[\bar{F}_{j 0}, \quad \bar{F}_{j 1}, \ldots, \bar{F}_{j s_{j}}\right]^{T} \in R^{s_{j}+1}, \\
\varpi(t) & =\left[1, f_{11}(t), \ldots, f_{1 s_{1}}(t), \ldots, 1, f_{j 1}(t), \ldots, f_{j s_{j}}(t), \ldots,\right. \\
& \left.1, f_{n 1}(t), \ldots, f_{n s_{n}}(t)\right]^{T}, \text { for } j=1, \ldots, n, \quad 15
\end{aligned}
$$

where $\nu_{0}(t)$ is a designed control signal, and $k_{\nu}$ is the actuator failure pattern parameter with $\xi$ and $\varpi(t)$ describing actuators and the types of failures.

For adaptive actuator failure compensation design, an assumption is given as: (A1) for the case that any up to $\bar{n}(\bar{n}<n)$ actuators fail, the remaining healthy actuators can still achieve the desired control objective. This assumption means that any $\bar{n}$ of the $n$ actuators may fail, and the parameter $k_{\nu}$ only takes one integer in the interval $[n-\bar{n}, n]$ to reflect the different failures.

Unparameterized failure model. Consider the incompletely parameterized failures. The mathematical model is given by:

$$
F_{j}(t)=\bar{F}_{j}(t)=\bar{F}_{j 0}+\sum_{\rho=1}^{s_{j}} \bar{F}_{j \rho} f_{j \rho}(t)+\bar{\delta}_{j}(t), \quad t \geq t_{j},(16)
$$

where $\bar{F}_{j 0}, \bar{F}_{j \rho}$ and $f_{j \rho}(t)$ are defined in (11), and $\bar{\delta}_{j}(t)$ is an unknown and unparameterizable but bounded term. In this case, the input of system (7)-(8) can be rewritten as

$$
F(t)=k_{\nu} \nu_{0}(t)+\xi^{T} \varpi(t)+\sum_{j=1}^{n-k_{\nu}} \bar{\delta}_{j}(t),
$$

where $\nu_{0}(t)$ is a designed control signal, $k_{\nu}, \xi$ and $\varpi(t)$ are defined in (13)-(15).

For the unparameterized failure case, it is assumed that Assumption (A1) holds for failure compensation design. The term $\bar{\delta}_{j}(t)$ is introduced to describe the nonparametric part of the failure, which can be changed to describe different failures. As $\bar{\delta}_{j}(t)=0,(16)$ is equivalent to the parametric failure (11).

Objective. The objective of this paper is to develop an adaptive failure compensation controller $\nu_{0}(t)$ for the above modeled high-speed trains (7)-(8) with unknown system parameters, unparameterizabel disturbances, and unknown actuator failures to guarantee the system stability and asymptotic tracking properties.

\section{Adaptive Controller for Healthy Actuators}

In this section, a backsteeping adaptive controller will be designed for the healthy trains to guarantee the stability of the closed-loop system and the state $x_{1}(t)$ to track the DistanceTo-Go (DTG) curve $x_{m}(t)$. The design procedure is given as follows:

Step 1: Denote the tracking error as

$$
z_{1}(t)=x_{1}(t)-x_{m}(t)
$$

and introduce

$$
z_{2}(t)=x_{2}(t)-\alpha_{1}(t)
$$

where $\alpha_{1}(t)$ is to be designed later. Then, from (7), it has

$$
\begin{aligned}
\dot{z}_{1}(t) & =\dot{x}_{1}(t)-\dot{x}_{m}(t) \\
& =z_{2}(t)+\alpha_{1}(t)-\dot{x}_{m}(t) .
\end{aligned}
$$

Choosing the design function $\alpha_{1}(t)$ as

$$
\alpha_{1}(t)=-r_{1} z_{1}(t)+\dot{x}_{m}(t), \quad r_{1}>0
$$

and considering the first partial positive definite function

$$
V_{1}=\frac{1}{2} z_{1}^{2}
$$


From (20) and (21), the time derivative of $V_{1}$ is given by:

$$
\begin{aligned}
\dot{V}_{1} & =z_{1}(t) \dot{z}_{1}(t) \\
& =z_{1}(t)\left(z_{2}(t)-r_{1} z_{1}(t)+\dot{x}_{m}(t)-\dot{x}_{m}(t)\right) \\
& =-r_{1} z_{1}^{2}(t)+z_{1}(t) z_{2}(t) .
\end{aligned}
$$

Step 2: From (19) and (8), it follows that

$$
\begin{aligned}
\dot{z}_{2}(t)= & \dot{x}_{2}(t)-\dot{\alpha}_{1}(t) \\
= & \sum_{i=1}^{l}\left(m_{i} \chi_{i}(t) F(t)-a_{i} \chi_{i}(t)-b_{i} \chi_{i}(t) x_{2}(t)\right. \\
& -c_{i} \chi_{i}(t) x_{2}^{2}(t)-g \vartheta_{i} \chi_{i}(t)-0.004 D_{i} \chi_{i}(t) \\
& \left.+\bar{d}_{i}(t) \chi_{i}(t)\right)-\dot{\alpha}_{1}(t) .
\end{aligned}
$$

Now, (20) and (24) can be viewed to be stabilized by $\alpha_{1}(t)$ given in (21) with respect to the Lyapunov function

$$
\begin{aligned}
V_{2}= & V_{1}+\frac{1}{2} z_{2}^{2}+\sum_{i=1}^{l} \frac{1}{2}\left(\Gamma_{a i}^{-1} \tilde{a}_{i}^{2}+\Gamma_{b i}^{-1} \tilde{b}_{i}^{2}+\Gamma_{c i}^{-1} \tilde{c}_{i}^{2}\right. \\
& \left.+\Gamma_{\vartheta i}^{-1} \tilde{\vartheta}_{i}^{2}+\Gamma_{D i}^{-1} \tilde{D}_{i}^{2}+\frac{m_{i}}{\Gamma_{m i}} \tilde{\rho}_{i}^{2}\right),
\end{aligned}
$$

where $\Gamma_{a i}, \Gamma_{b i}, \Gamma_{c i}, \Gamma_{\vartheta i}, \Gamma_{D i}$ and $\Gamma_{m i}$ are positive constants, $\tilde{a}_{i}=a_{i}-\hat{a}_{i}(t), \tilde{b}_{i}=b_{i}-\hat{b}_{i}(t), \tilde{c}_{i}=c_{i}-\hat{c}_{i}(t), \tilde{\vartheta}_{i}=\vartheta_{i}-\hat{\vartheta}_{i}(t)$, $\tilde{D}_{i}=D_{i}-\hat{D}_{i}(t)$, with $\hat{a}_{i}(t), \hat{b}_{i}(t), \hat{c}_{i}(t), \hat{\vartheta}_{i}(t), \hat{D}_{i}(t)$ being the estimates of $a_{i}, b_{i}, c_{i}, \vartheta_{i}$, and $D_{i} . \tilde{\rho}_{i}=\rho_{i}-\hat{\rho}_{i}(t), \hat{\rho}_{i}(t)$ is the estimate of $\rho_{i}=\frac{1}{m_{i}}$. The associate adaptive laws used to estimate these parameters will be provided later.

Using (8), the time derivative of $V_{2}$ is

$$
\begin{aligned}
\dot{V}_{2}= & -r_{1} z_{1}^{2}(t)+z_{1}(t) z_{2}(t)+z_{2} \dot{z}_{2}(t)+\sum_{i=1}^{l}\left(\Gamma_{a i}^{-1} \tilde{a}_{i}(t) \dot{\tilde{a}}_{i}(t)\right. \\
& +\Gamma_{b i}^{-1} \tilde{b}_{i}(t) \dot{\tilde{b}}_{i}(t)+\Gamma_{c i}^{-1} \tilde{c}_{i}(t) \dot{\tilde{c}}_{i}(t)+\Gamma_{\vartheta i}^{-1} \tilde{\vartheta}_{i}(t) \dot{\tilde{\vartheta}}_{i}(t) \\
& \left.+\Gamma_{D i}^{-1} \tilde{D}_{i}(t) \dot{\tilde{D}}_{i}(t)+\frac{m_{i}}{\Gamma_{m i}} \tilde{\rho}_{i}(t) \dot{\tilde{\rho}}_{i}(t)\right) \\
= & -r_{1} z_{1}^{2}(t)+z_{2}(t)\left(x_{1}(t)-x_{m}(t)\right) \\
& +z_{2}(t) \sum_{i=1}^{l}\left(m_{i} \chi_{i}(t) F(t)-\hat{a}_{i} \chi_{i}(t)-\hat{b}_{i} \chi_{i}(t) x_{2}(t)\right. \\
& \left.-\hat{c}_{i} \chi_{i}(t) x_{2}^{2}(t)-g \hat{\vartheta}_{i} \chi_{i}(t)-0.004 \hat{D}_{i} \chi_{i}(t)+\bar{d}_{i}(t) \chi_{i}(t)\right) \\
& +z_{2}(t)\left(r_{1}\left(\dot{x}_{1}(t)-\dot{x}_{m}(t)\right)-\ddot{x}_{m}(t)\right) \\
& +\sum_{i=1}^{l}\left(\Gamma_{a i}^{-1} \tilde{a}_{i}(t)\left(\dot{\hat{a}}_{i}(t)+\Gamma_{a i} z_{2}(t) \chi_{i}(t)\right)\right. \\
& +\Gamma_{b i}^{-1} \tilde{b}_{i}(t)\left(\dot{\hat{b}}_{i}(t)+\Gamma_{b i} z_{2}(t) \chi_{i}(t) x_{2}(t)\right) \\
& +\Gamma_{c i}^{-1} \tilde{c}_{i}(t)\left(\dot{\hat{c}}_{i}(t)+\Gamma_{c i} z_{2}(t) \chi_{i}(t) x_{2}^{2}(t)\right) \\
& +\Gamma_{\vartheta i}^{-1} \tilde{\vartheta}_{i}(t)\left(\dot{\hat{\vartheta}}_{i}(t)+\Gamma_{\vartheta i} z_{2}(t) \chi_{i}(t) g\right) \\
& +\Gamma_{D i}^{-1} \tilde{D}_{i}(t)\left(\dot{\hat{D}}_{i}(t)+0.004 \Gamma_{D i} z_{2}(t) \chi_{i}(t)\right) \\
& \left.+\frac{m_{i}}{\Gamma_{m i}} \tilde{\rho}_{i}(t) \dot{\tilde{\rho}}_{i}(t)\right) .
\end{aligned}
$$

Choose the adaptive laws for $\hat{a}_{i}(t), \hat{b}_{i}(t), \hat{c}_{i}(t), \hat{\vartheta}_{i}(t), \hat{D}_{i}(t)$

$$
\begin{aligned}
& \dot{\hat{\hat{a}}}_{i}(t)=-\Gamma_{a i} z_{2}(t) \chi_{a i}(t), \\
& \dot{\hat{b}}_{i}(t)=-\Gamma_{b i} z_{2}(t) \chi_{b i}(t) x_{2}(t), \\
& \dot{\hat{c}}_{i}(t)=-\Gamma_{c i} z_{2}(t) \chi_{c i}(t) x_{2}^{2}(t), \\
& \dot{\hat{\vartheta}}_{i}(t)=-\Gamma_{\vartheta i} z_{2}(t) \chi_{\vartheta i}(t) g, \\
& \dot{\hat{D}}_{i}(t)=-0.004 \Gamma_{D i} z_{2}(t) \chi_{D i}(t) .
\end{aligned}
$$

Then the time derivative of $V_{2}$ can be expressed as

$$
\begin{aligned}
\dot{V}_{2}= & -r_{1} z_{1}^{2}(t)+z_{2}(t)\left(\eta(t)+\sum_{i=1}^{l}\left(m_{i} \chi_{i}(t) F(t)\right.\right. \\
& \left.\left.-\zeta_{i}(t) \chi_{i}(t)+\bar{d}_{i}(t) \chi_{i}(t)\right)\right)+\sum_{i=1}^{l} \frac{m_{i}}{\Gamma_{m i}} \tilde{\rho}_{i}(t) \dot{\tilde{\rho}}_{i}(t)
\end{aligned}
$$

where $\eta(t)$ and $\zeta_{i}(t)$ are given as

$$
\begin{aligned}
\eta(t)= & x_{1}(t)-x_{m}(t)+r_{1}\left(\dot{x}_{1}(t)-\dot{x}_{m}(t)\right)-\ddot{x}_{m}(t), \\
\zeta_{i}(t)= & \hat{a}_{i} \chi_{a i}(t)+\hat{b}_{i} \chi_{b i}(t) x_{2}(t)+\hat{c}_{i} \chi_{c i}(t) x_{2}^{2}(t) \\
& +g \hat{\vartheta}_{i} \chi_{\vartheta i}(t)+0.004 \hat{D}_{i} \chi_{D i}(t) .
\end{aligned}
$$

Considering that the indicator function $\chi_{m i}(t)$ can only be $0-1$ function, the expression of $\dot{V}_{2}$ can be rewritten as

$$
\begin{aligned}
\dot{V}_{2}= & -r_{1} z_{1}^{2}(t)+z_{2}(t) \sum_{i=1}^{l} m_{i} \chi_{m i}(t)\left(F(t)-\rho_{i} \zeta_{i}(t)\right. \\
& \left.+\rho_{i} \bar{d}_{i}(t) \chi_{\bar{d} i}(t)+\rho_{i} \eta(t)\right)+\sum_{i=1}^{l} \frac{m_{i}}{\Gamma_{m i}} \tilde{\rho}_{i}(t) \dot{\tilde{\rho}}_{i}(t) \\
= & -r_{1} z_{1}^{2}(t)+z_{2}(t) \sum_{i=1}^{l} m_{i} \chi_{m i}(t)\left(F(t)-\rho_{i} \zeta_{i}(t)\right. \\
& \left.+\rho_{i} \bar{d}_{i}(t) \chi_{\bar{d} i}(t)+\rho_{i} \eta(t)+\rho_{i}(t) r_{2} z_{2}(t)\right) \\
& -r_{2} z_{2}^{2}(t)+\sum_{i=1}^{l} \frac{m_{i}}{\Gamma_{m i}} \tilde{\rho}_{i}(t) \dot{\tilde{\rho}}_{i}(t),
\end{aligned}
$$

where $r_{2}>0$.

The controller $F(t)$ is chosen as

$$
\begin{aligned}
F(t)= & \sum_{i=1}^{l} \chi_{m i}(t)\left(\nu_{d i}(t) \chi_{\bar{d} i}(t)+\hat{\rho}_{i}(t) \zeta_{i}(t)\right. \\
& \left.-\hat{\rho}_{i}(t) \eta(t)-\hat{\rho}_{i}(t) r_{2} z_{2}(t)\right)
\end{aligned}
$$

where $\nu_{d i}(t)$ is designed to compensate the unmodeled disturbance $d_{i}(t)$ for stability and tracking, $\hat{\rho}_{i}(t)$ is updated from the adaptive law as follows:

$$
\begin{aligned}
\nu_{d i}(t) & =-\frac{1}{\lambda_{i}^{0}} \epsilon_{i}^{0} \operatorname{sgn}\left[z_{2}(t)\right], \\
\dot{\hat{\rho}}_{i}(t) & =-\Gamma_{m i} z_{2}(t)\left(\zeta_{i}(t)-\eta(t)-r_{2} z_{2}(t)\right) \chi_{m i}(t),
\end{aligned}
$$

with $\rho_{m}^{0}$ being a known lower bound on $m_{i}: m_{i} \geq \rho_{m}^{0}$, and $\lambda_{i}^{0}$ being a design parameter such that $\lambda_{i}^{0} \geq d_{i}^{0}$. 
Using the controller (35), with (36) and (37), $\dot{V}_{2}$ can be rewritten by

$$
\begin{aligned}
\dot{V}_{2}= & -r_{1} z_{1}^{2}(t)-r_{2} z_{2}^{2}(t)+\sum_{i=1}^{l}\left(-\frac{m_{i}}{\rho_{m}^{0}} \lambda_{i}^{0}\left|z_{2}(t)\right| \chi_{\bar{d} i}(t)\right. \\
& \left.+z_{2}(t) \bar{d}_{i}(t) \chi_{\bar{d} i}(t)\right) \\
\leq & -r_{1} z_{1}^{2}(t)-r_{2} z_{2}^{2}(t)
\end{aligned}
$$

Stability analysis. Since $\dot{V}_{2} \leq-r_{1} z_{1}^{2}(t)-r_{2} z_{2}^{2}(t) \leq 0$, all the variables $z_{1}(t)=x_{1}(t)-x_{m}(t), z_{2}(t)=x_{2}(t)-\alpha_{1}(t)$, $\xi-\hat{\xi}(t), a_{i}-\hat{a}_{i}(t), b_{i}-\hat{b}_{i}(t), c_{i}-\hat{c}_{i}(t), \vartheta_{i}-\hat{\vartheta}_{i}(t), D_{i}-\hat{D}_{i}(t)$, $\rho_{i}-\hat{\rho}_{i}(t)$, are bounded, and so are $z_{1}(t), \hat{\xi}(t), \hat{a}_{i}(t), \hat{b}_{i}(t)$, $\hat{c}_{i}(t), \hat{\vartheta}_{i}(t), \hat{D}_{i}(t)$, and $\hat{\rho}_{i}(t)$. From $(21), \alpha_{1}(t)$ is bounded, so is $x_{2}(t)$. Then, from (35), the boundedness of the control $F(t)$ is ensured. Thus, all signals in the closed-loop system are bounded.

Since $V_{2}$ is bounded, $\int_{0}^{\infty} z_{1}^{2}(\tau) d \tau<\infty$ and $\int_{0}^{\infty} z_{2}^{2}(\tau) d \tau<$ $\infty$. According to $(20), \dot{z}_{1}(t)$ is bounded. It shows that $\lim _{t \rightarrow \infty} z_{1}(t)=0$, which implies that $\lim _{t \rightarrow \infty}\left(x_{1}(t)-\right.$ $\left.x_{m}(t)\right)=0$.

It should be note that the control signal in (36) is not continuous. Thus $\dot{V}_{2}$ is negative with discontinuous right-handside. In this case, the system solution is defined in the Filippov sense [25], [26].

Now, we obtain the following adaptive controller design method:

Theorem 1: The adaptive controller (35), with the adaptive scheme (27)-(31) and (36)-(37) applied to the system (7)-(8), guarantees that all the closed-loop signals are bounded and the tracking error $e(t)=x_{1}(t)-x_{m}(t)$ satisfies $\lim _{t \rightarrow \infty} e(t)=0$.

From (21), $\dot{\alpha}(t)$ is bounded, due to the bounded $\dot{z}_{1}(t)$ and $\dot{x}_{m}(t)$. According to (24), $\dot{z}_{2}(t)$ is bounded. It shows that $\lim _{t \rightarrow \infty} z_{2}(t)=0$. Further, with bounded $x_{2}(t)$ and $\dot{x}_{m}(t)$, it follows that $\lim _{t \rightarrow \infty} x_{2}(t)-\dot{x}_{m}(t)=0$, which implies that the $x_{2}(t)$ can track $\dot{x}_{m}(t)$. For high-speed train, $\dot{x}_{m}(t)$ represents the desired speed of the train. Therefore, the adaptive controller (35) can achieve both the distance and speed tracking, simultaneously. To design the tracking curve, it is necessary to consider the constraint for distance and speed of the train under the different operating conditions.

\section{Adaptive Actuator Failure Compensation CONTROLLER}

In this section, a failure compensation controller will be designed to guarantee the closed-loop system to be stable and the state $x_{1}(t)$ to track the desired curve $x_{m}(t)$ in the presence of actuator failures. The parameters of the train dynamic model are unknown constants with known bounds on disturbance, but uncertain actuator failures may occur. The design procedure is described as follows:

Step 1: This step is the same as that for healthy case in Section III. The tracking error $z_{1}(t)$ and the parameter $z_{2}(t)$ are defined in (18) and (19), respectively. The first partial positive definite function $V_{1}$ is chosen as (22).
Step 2: From $z_{2}(t)=x_{2}(t)-\alpha_{1}(t),(8)$ and (13),

$$
\begin{aligned}
\dot{z}_{2}(t)= & \sum_{i=1}^{l}\left(m_{i} \chi_{m i}(t)\left(k_{\nu} \nu_{0}(t)+\xi^{T} \varpi(t)\right)-a_{i} \chi_{a i}(t)\right. \\
& -b_{i} \chi_{b i}(t) x_{2}(t)-c_{i} \chi_{c i}(t) x_{2}^{2}(t)-g \vartheta_{i} \chi_{\vartheta i}(t) \\
& \left.-0.004 D_{i} \chi_{D i}(t)+\bar{d}_{i}(t) \chi_{\bar{d} i}(t)\right)-\dot{\alpha}_{1}(t) .
\end{aligned}
$$

For fault compensation problem, the dynamics of $z_{1}(t)$ and $z_{2}(t)$ can be viewed to be stabilized by $\alpha_{1}(t)$ given in (21) with respect to the following candidate Lyapunov function

$$
\begin{aligned}
V_{2}= & V_{1}+\frac{1}{2} z_{2}^{2}+\sum_{i=1}^{l} \frac{1}{2} \frac{m_{i}}{\Gamma_{k \nu}} \tilde{\rho}_{k \nu}^{2}+\sum_{i=1}^{l} \frac{m_{i}}{2} \Gamma_{\xi}^{-1} \tilde{\xi}^{T} \tilde{\xi} \\
& +\sum_{i=1}^{l} \frac{1}{2}\left(\Gamma_{a i}^{-1} \tilde{a}_{i}^{2}+\Gamma_{b i}^{-1} \tilde{b}_{i}^{2}+\Gamma_{c i}^{-1} \tilde{c}_{i}^{2}+\Gamma_{\vartheta i}^{-1} \tilde{\vartheta}_{i}^{2}\right. \\
& \left.+\Gamma_{D i}^{-1} \tilde{D}_{i}^{2}+\frac{m_{i}}{\Gamma_{m i}} \tilde{\rho}_{i}^{2}\right),
\end{aligned}
$$

where $V_{1}$ is defined in (22), $\Gamma_{k \nu}, \Gamma_{\xi}, \Gamma_{a i}, \Gamma_{b i}, \Gamma_{c i}, \Gamma_{\vartheta i}, \Gamma_{D i}$ and $\Gamma_{m i}$ are positive constants, $\tilde{\xi}=\xi-\hat{\xi}(t), \tilde{a}_{i}=a_{i}-$ $\hat{a}_{i}(t), \tilde{b}_{i}=b_{i}-\hat{b}_{i}(t), \tilde{c}_{i}=c_{i}-\hat{c}_{i}(t), \tilde{\vartheta}_{i}=\vartheta_{i}-\hat{\vartheta}_{i}(t)$, $\tilde{D}_{i}=D_{i}-\hat{D}_{i}(t)$, with $\hat{\xi}(t), \hat{a}_{i}(t), \hat{b}_{i}(t), \hat{c}_{i}(t), \hat{\vartheta}_{i}(t), \hat{D}_{i}(t)$ being the estimates of $\xi, a_{i}, b_{i}, c_{i}, \vartheta_{i}, D_{i}$, respectively, and $\tilde{\rho}_{k \nu}=\rho_{k \nu}-\hat{\rho}_{k \nu}(t), \tilde{\rho}_{i}=\rho_{i}-\hat{\rho}_{i}(t)$, with $\hat{\rho}_{k \nu}(t)$ and $\hat{\rho}_{i}(t)$ being the estimate of $\rho_{k \nu}=\frac{1}{k_{\nu}}$ and $\rho_{i}=\frac{1}{m_{i} k_{\nu}}$, respectively.

It should be noted that there are two types (sets) of time intervals: one set of known time intervals during which the system parameters are constant and unknown, and one set of unknown time intervals during which the actuator failure parameters are constant and unknown (as the failure pattern is fixed). To deal with the first type of known time intervals, we have introduced the indicator functions $\chi_{\varrho i}(t)$ to expand the system parametrization to include all possible piecewise values of system parameters, that is, the parameters $a_{i}, b_{i}, c_{i}$, $\vartheta_{i}, D_{i}$ and $\frac{1}{m_{i}}$ (related with $\rho_{i}$ ) are constant for all such time intervals. To deal with the second type of unknown time intervals, let such time intervals be $\left(T_{p}, T_{p+1}\right), p=0,1, \ldots, \bar{M}$, that is, for $t \in\left(T_{p}, T_{p+1}\right)$, the actuator failure pattern is fixed and the parameters in (13) are constant, which implies that the parameters $\xi$ and $k \nu$ in (40) are constant. It should be noted that $\rho_{i}=\frac{1}{m_{i} k_{\nu}}$ changes with the indicator function $\chi_{m i}(t)$ and the failure pattern. Let $\left\{T_{q}\right\}_{q=1}^{\infty}$ denote the known time instants at which (7)-(8) switches between different modes. Without loss of generality, we assume that $T_{p} \in\left(T_{q}, T_{q+1}\right)$ and $T_{p+1}>T_{q+1}$. Then, the positive definite function $V_{2}$ is continuous and differentiable on the time intervals $\left(T_{q}, T_{p}\right)$.

The adaptive laws for $\hat{a}_{i}(t), \hat{b}_{i}(t), \hat{c}_{i}(t), \hat{\vartheta}_{i}(t)$, and $\hat{D}_{i}(t)$ are chosen the same as that of health case (27)-(31). $\hat{\xi}(t)$ is updated by

$$
\dot{\hat{\xi}}(t)=\Gamma_{\xi} z_{2}(t) \varpi(t),
$$

Then, From equations (21), (23) and (39), the time derivative 
of $V_{2}$ is

$$
\begin{aligned}
\dot{V}_{2}= & -r_{1} z_{1}^{2}(t)+z_{2}(t) \sum_{i=1}^{l} m_{i} k_{\nu} \chi_{m i}(t)\left(\nu_{0}(t)+\rho_{k \nu} \hat{\xi}^{T}(t) \varpi(t)\right. \\
& \left.-\rho_{i} \zeta_{i}(t)+\rho_{i} \bar{d}_{i}(t) \chi_{\bar{d} i}(t)+\rho_{i} \eta(t)+\rho_{i} r_{2} z_{2}(t)\right) \\
& -r_{2} z_{2}^{2}(t)+\sum_{i=1}^{l} \frac{m_{i}}{\Gamma_{k \nu}} \tilde{\rho}_{k \nu}(t) \dot{\tilde{\rho}}_{k \nu}(t)+\sum_{i=1}^{l} \frac{m_{i}}{\Gamma_{m i}} \tilde{\rho}_{i}(t) \dot{\tilde{\rho}}_{i}(t),
\end{aligned}
$$

where $r_{2}>0, \eta(t)$ and $\zeta_{i}(t)$ are given as (32) and (33).

The control signal $\nu_{0}(t)$ is designed as

$$
\begin{aligned}
\nu_{0}(t)= & -\hat{\rho}_{k \nu}(t) \hat{\xi}^{T}(t) \varpi(t)+\sum_{i=1}^{l} \chi_{m i}(t)\left(\nu_{d i}(t)\right. \\
& \left.+\hat{\rho}_{i}(t) \zeta_{i}(t)-\hat{\rho}_{i}(t) \eta(t)-\hat{\rho}_{i}(t) r_{2} z_{2}(t)\right),
\end{aligned}
$$

where $\hat{\rho}_{i}(t)$ is given as (37), $\nu_{d i}(t)$ is designed to compensate unmodeled disturbance $d_{i}(t)$ to guarantee the system stability and tracking performance, and $\hat{\rho}_{k \nu}(t)$ is updated from the adaptive law

$$
\begin{aligned}
& \nu_{d i}(t)=-\frac{1}{\rho_{i}^{0}} \lambda_{i}^{0} \operatorname{sgn}\left[z_{2}(t)\right], \\
& \dot{\hat{\rho}}_{k \nu}(t)=-\Gamma_{k \nu} z_{2}(t) \hat{\xi}^{T}(t) \varpi(t),
\end{aligned}
$$

with $\rho_{i}^{0}$ being a known lower bound on $m_{i}: m_{i} k_{\nu} \geq \rho_{i}^{0}$, and $\lambda_{i}^{0}$ being a design parameter such that $\lambda_{i}^{0} \geq d_{i}^{0}$.

The controller (42), with the signal $\nu_{d i}(t)$ defined in (43) and the adaptive control laws in (37) and (44), leads to

$$
\begin{aligned}
\dot{V}_{2}= & -r_{1} z_{1}^{2}(t)-r_{2} z_{2}^{2}(t)+\sum_{i=1}^{l}\left(-\frac{m_{i} k_{\nu}}{\rho_{i}^{0}} \lambda_{i}^{0}\left|z_{2}(t)\right| \chi_{\bar{d} i}(t)\right. \\
& \left.+z_{2}(t) \bar{d}_{i}(t) \chi_{\bar{d} i}(t)\right) \\
\leq & -r_{1} z_{1}^{2}(t)-r_{2} z_{2}^{2}(t)
\end{aligned}
$$

It should be noted that the values of piecewise constant parameters, also with the amplitudes of failure parameters could change, during the system operation. We denote $\left(T_{p}, T_{p+1}\right)$, $p=0,1, \ldots, \bar{M}$, with $T_{0}=0$, be time intervals, when the actuator failure pattern is fixed, which means that the actuators only fail at time $T_{p}$, for $p=0,1, \ldots, \bar{M}$. Under Assumption (A1), $T_{\bar{M}+1}=\infty$. Meanwhile, let $\left\{T_{q}\right\}_{q=1}^{\infty}$ denote the known time instants at which (7)-(8) switches between different modes. That is, the unknown parameters $\rho_{k \nu}$ and $\xi$ related to the failure parameters $k_{\nu}$ and $\xi$, change their values, at time $T_{p}$, $p=0,1, \ldots, \bar{M}$, while the unknown system parameters $a_{i}, b_{i}$, $c_{i}, \vartheta_{i}$, and $D_{i}$ change their values, at time $T_{q}, q=1, \ldots, \infty$, and $\rho_{i}$ (related with $m_{i}$ and $k_{\nu}$ ) changes its value, at time $T_{q}$ and $T_{p}$, for $q=1, \ldots, \infty$ and $p=0,1, \ldots, \bar{M}$.

Stability analysis. For the function $V_{2}$ in (40), the term containing $\tilde{\rho}_{k \nu}$ and $\tilde{\xi}$ about the failures is different from the term in (25) (last term, containing $\tilde{a}_{i}, \tilde{b}_{i}, \tilde{c}_{i}, \tilde{\vartheta}_{i}, \tilde{D}_{i}$ and $\tilde{\rho}_{i}$ ) about the model parameters, because the switches of the failures are achieved via the tracking error based adaptive laws instead of the indicator functions $\chi_{\varrho}(t)$ in (6). Due to the piecewise constant parameters $\rho_{k \nu}, \xi, a_{i}, b_{i}, c_{i}, \vartheta_{i}, D_{i}$ and $\rho_{i}$,
$V_{2}(\cdot)$ as a function of $t$ is not continuous. With the estimation errors $z_{1}(t), z_{2}(t)$ and the adaptive laws in (27)-(31), (37), (41) and (44), the time derivative of $V_{2}$ for $t \in\left(T_{q}, T_{p}\right)$, $p=0,1, \ldots, \bar{M}$, becomes

$$
\dot{V}_{2} \leq-r_{1} z_{1}^{2}(t)-r_{2} z_{2}^{2}(t) .
$$

Since there are only a finite number of failures in the system, $V_{2}\left(T_{\bar{M}}\right)$ is finite, and from

$$
\dot{V}_{2} \leq-r_{1} z_{1}^{2}(t)-r_{2} z_{2}^{2}(t) \leq 0
$$

it follows that all the variables $z_{1}(t)=x_{1}(t)-x_{m}(t)$, $z_{2}(t)=x_{2}(t)-\alpha_{1}(t), \xi-\hat{\xi}(t), a_{i}-\hat{a}_{i}(t), b_{i}-\hat{b}_{i}(t), c_{i}-\hat{c}_{i}(t)$, $\vartheta_{i}-\hat{\vartheta}_{i}(t), D_{i}-\hat{D}_{i}(t), \rho_{k \nu}-\hat{\rho}_{k \nu}(t)$, and $\rho_{i}-\hat{\rho}_{i}(t)$ are bounded, and so are $z_{1}(t), \hat{\xi}(t), \hat{a}_{i}(t), \hat{b}_{i}(t), \hat{c}_{i}(t), \hat{\vartheta}_{i}(t)$, $\hat{D}_{i}(t), \hat{\rho}_{k \nu}(t)$ and $\hat{\rho}_{i}(t)$. From $(21), \alpha_{1}(t)$ is bounded, so is $x_{2}(t)$. Then, with the failure compensation controller (42), the boundedness of $\nu_{0}(t)$ is ensured. Thus, all signal in the closedloop system are bounded. Further, (47) implies $z_{1}(t) \in L_{2}$ and so $\lim _{t \rightarrow \infty} z_{1}(t)=0$.

It should be noted that the control signal in (43) is not continuous, then $\dot{V}_{2}$ is negative with discontinuous right-handside. The system solution is defined in the Filippov sense [25], [26]. The performance of the adaptive controller to obtain the stability and tracking can be summarized as follows:

Theorem 2: The adaptive failure compensation controller (42), with the adaptive scheme (27)-(31), (37), (41) and (44) applied to the system (7)-(8) with actuator failures (13)-(15), guarantees that all the closed-loop signals are bounded and the tracking error $e(t)=x_{1}(t)-x_{m}(t)$ satisfies $\lim _{t \rightarrow \infty} e(t)=0$.

Similar to the convergence discussion in Section III, we have $\lim _{t \rightarrow \infty}\left(x_{2}(t)-\dot{x}_{m}(t)\right)=0$. The designed adaptive compensation controller (42) can achieve the distance and speed tracking, simultaneously. The proposed adaptive compensation controller is designed effectively for the complete parameterized high-speed train faulty model with known bounds of disturbance. For the following sections, the more general model with the different mathematical formulations of the unparameterizable parameters will be discussed.

\section{Design For UnParameterizable ACtuator FAILURES}

Recall the incompletely parameterized failure model (16) and (17) in Section II. In this section, the more general actuator failure problem will be considered.

The design procedure is similar as that of the parameterizable case in Section IV. Due to the unparameterizable actuator failures, the controller $\nu_{0}(t)$ is designed as

$$
\begin{aligned}
\nu_{0}(t)= & -\hat{\rho}_{k \nu}(t) \hat{\xi}(t) \varpi(t)+\sum_{j=1}^{n-k_{\nu}} \nu_{s j}(t)+\sum_{i=1}^{l} \chi_{m i}(t)\left(\nu_{d i}(t)\right. \\
& \left.+\hat{\rho}_{i}(t) \zeta_{i}(t)-\hat{\rho}_{i}(t) \eta(t)-\hat{\rho}_{i}(t) r_{2} z_{2}(t)\right)
\end{aligned}
$$

where $\nu_{s j}(t)$ and $\nu_{d i}(t)$ are designed to compensate unknown $\bar{\delta}_{j}(t)$ and $\bar{d}_{i}(t)$ for stability and tracking; $\eta(t)$ and $\zeta_{i}(t)$ are defined in (32) and (33) with the estimates $\hat{a}_{i}(t), \hat{b}_{i}(t), \hat{c}_{i}(t)$, 
$\hat{\vartheta}_{i}(t), \hat{D}_{i}(t)$ being updated by the adaptive laws in (27)-(31); $\hat{\rho}_{i}(t), \hat{\xi}(t)$ and $\hat{\rho}_{k \nu}(t)$ are given by the adaptive laws (37), (41) and (44).

The design signals $\nu_{s j}(t)$ and $\nu_{d i}(t)$ are designed:

$$
\begin{aligned}
& \nu_{s j}(t)=-\frac{1}{\rho_{k \nu}^{0}} \delta_{j}^{0} \operatorname{sgn}\left[z_{2}(t)\right], \\
& \nu_{d i}(t)=-\frac{1}{\rho_{i}^{0}} \lambda_{i}^{0} \operatorname{sgn}\left[z_{2}(t)\right],
\end{aligned}
$$

where $\rho_{k_{\nu}}^{0}$ and $\rho_{i}^{0}$ are known lower bounds on $k_{\nu}$ and $m_{i} k_{\nu}$, respectively: $k_{\nu} \geq \rho_{k \nu}^{0}, m_{i} k_{\nu} \geq \rho_{i}^{0}$, and $\delta_{j}^{0} \geq\left|\bar{\delta}_{j}(t)\right|, \lambda_{i}^{0}$ is a design parameter such that $\lambda_{i}^{0} \geq d_{i}^{0}$.

Using the Lyapunov function (40), the above adaptive control scheme leads to

$$
\begin{aligned}
\dot{V}_{2}= & -r_{1} z_{1}^{2}(t)-r_{2} z_{2}^{2}(t)+\sum_{i=1}^{l} \sum_{j=1}^{n-k_{\nu}}\left(m_{i} z_{2}(t) \bar{\delta}_{j}(t) \chi_{m i}(t)\right. \\
& +z_{2}(t) \bar{d}_{i}(t) \chi_{\bar{d} i}(t)-\frac{m_{i} k_{\nu}}{\rho_{k \nu}^{0}} \delta_{j}^{0}\left|z_{2}(t)\right| \chi_{m i}(t) \\
& \left.-\frac{m_{i} k_{\nu}}{\rho_{i}^{0}} \lambda_{i}^{0}\left|z_{2}(t)\right| \chi_{\bar{d} i}(t)\right) \\
\leq & -r_{1} z_{1}^{2}(t)-r_{2} z_{2}^{2}(t) .
\end{aligned}
$$

Stability analysis. Due to the finite number of failures in the system, $V_{2}\left(T_{\bar{M}}\right)$ is finite. From

$$
\dot{V}_{2}=-r_{1} z_{1}^{2}(t)-r_{2} z_{2}^{2}(t) \leq 0,
$$

the variables $z_{1}(t)=x_{1}(t)-x_{m}(t), z_{2}(t)=x_{2}(t)-\alpha_{1}(t)$, $\xi-\hat{\xi}(t), a_{i}-\hat{a}_{i}(t), b_{i}-\hat{b}_{i}(t), c_{i}-\hat{c}_{i}(t), \vartheta_{i}-\hat{\vartheta}_{i}(t), D_{i}-\hat{D}_{i}(t)$, $\rho_{k \nu}-\hat{\rho}_{k \nu}(t), \rho_{i}-\hat{\rho}_{i}(t)$, are bounded, and so are $z_{1}(t), \hat{\xi}(t)$, $\hat{a}_{i}(t), \hat{b}_{i}(t), \hat{c}_{i}(t), \hat{\vartheta}_{i}(t), \hat{D}_{i}(t), \hat{\rho}_{k \nu}(t)$ and $\hat{\rho}_{i}(t)$. Then, with the structure of the failure compensation controller (48), the boundedness of $\nu_{0}(t)$ is ensured. Further, (52) implies $z_{1}(t) \in$ $L_{2}$ and so $\lim _{t \rightarrow \infty} z_{1}(t)=0$. The system solution is defined in the Filippov sense [25], [26].

For the unparameterizable actuator failures, we have the following conclusion:

Theorem 3: The adaptive failure compensation controller (48), with the signals (32)-(33) and (49)-(50), and adaptive scheme (27)-(31), (37), (41), and (44) applied to the system (7)-(8) under actuator failures (16), guarantees that all the closed-loop signals are bounded and the tracking error $e(t)=x_{1}(t)-x_{m}(t)$ satisfies $\lim _{t \rightarrow \infty} e(t)=0$.

Similar to the convergence discussion in Section III, we have that $\lim _{t \rightarrow \infty}\left(x_{2}(t)-\dot{x}_{m}(t)\right)=0$. For the unparameterizable failure case considered here, there are two type parameter variations caused by either actuator failure or system mode changes, also the disturbance and unparameterizable failure term. The proposed controller (48) is parameterized, so that both parameter variations and unparameterizable terms can be dealt with. To handle the unparameterizable terms from disturbances and failures, the signals $\nu_{s j}(t)$ and $\nu_{d i}(t)$ in (49) and (50), are employed to guarantee the tracking performance via the use of a piecewise Lyapunov function $V_{2}$.

\section{Design with UnKnown Disturbance Bounds}

The disturbances considered in the previous sections, are bounded with known bounds. If the disturbance bounds are unknown, some methods should be taken to estimate the bounds. In this section, we will propose a failure compensation controller for the case that the disturbance bounds are unknown.

The some design steps are same as that for the parameterizable case in Section III. Due to the unknown disturbance bounds, Lyapunov function $V_{2}$ should be changed and chosen as:

$$
\begin{aligned}
V_{2}= & V_{1}+\frac{1}{2} z_{2}^{2}+\sum_{i=1}^{l} \frac{1}{2} \frac{m_{i}}{\Gamma_{k \nu}} \tilde{\rho}_{k \nu}^{2}+\sum_{i=1}^{l} \frac{m_{i}}{2} \Gamma_{\xi}^{-1} \tilde{\xi}^{T} \tilde{\xi} \\
& +\sum_{i=1}^{l} \frac{1}{2}\left(\Gamma_{a i}^{-1} \tilde{a}_{i}^{2}+\Gamma_{b i}^{-1} \tilde{b}_{i}^{2}+\Gamma_{c i}^{-1} \tilde{c}_{i}^{2}+\Gamma_{\vartheta i}^{-1} \tilde{\vartheta}_{i}^{2}\right. \\
& \left.+\Gamma_{D i}^{-1} \tilde{D}_{i}^{2}+\Gamma_{d_{i}^{0}}^{-1}\left(\tilde{\lambda}_{i}^{0}\right)^{2}+\frac{m_{i}}{\Gamma_{m i}} \tilde{\rho}_{i}^{2}\right),
\end{aligned}
$$

where $V_{1}$ is defined in (22), $\Gamma_{k \nu}, \Gamma_{\xi}, \Gamma_{a i}, \Gamma_{b i}, \Gamma_{c i}, \Gamma_{\vartheta i}, \Gamma_{D i}$, $\Gamma_{m i}$ and $\Gamma_{d_{i}^{0}}$ are positive constants, $\tilde{\xi}=\xi-\hat{\xi}(t), \tilde{a}_{i}=a_{i}-$ $\hat{a}_{i}(t), \tilde{b}_{i}=b_{i}-\hat{b}_{i}(t), \tilde{c}_{i}=c_{i}-\hat{c}_{i}(t), \tilde{\vartheta}_{i}=\vartheta_{i}-\hat{\vartheta}_{i}(t)$, $\tilde{D}_{i}=D_{i}-\hat{D}_{i}(t), \tilde{\lambda}_{i}^{0}=\lambda_{i}^{0}-\hat{\lambda}_{i}^{0}(t)$ with $\hat{\xi}(t), \hat{a}_{i}(t), \hat{b}_{i}(t), \hat{c}_{i}(t)$, $\hat{\vartheta}_{i}(t), \hat{D}_{i}(t), \hat{\lambda}_{i}^{0}(t)$ being the estimates of $\xi, a_{i}, b_{i}, c_{i}, \vartheta_{i}, D_{i}$, and $\lambda_{i}^{0}$, respectively, and $\tilde{\rho}_{k \nu}=\rho_{k \nu}-\hat{\rho}_{k \nu}(t), \tilde{\rho}_{i}=\rho_{i}-\hat{\rho}_{i}(t)$, with $\hat{\rho}_{k \nu}(t)$ and $\hat{\rho}_{i}(t)$ being the estimate of $\rho_{k \nu}=\frac{1}{k_{\nu}}$ and $\rho_{i}=\frac{1}{m_{i} k_{\nu}}$, respectively. $\lambda_{i}^{0}$ is an unknown parameter such that $\lambda_{i}^{0} \geq d_{i}^{0}$.

The adaptive laws for $\hat{a}_{i}(t), \hat{b}_{i}(t), \hat{c}_{i}(t), \hat{\vartheta}_{i}(t), \hat{D}_{i}(t)$ and $\hat{\xi}(t)$ are given as (27)-(31) and (41). $\hat{\lambda}_{i}^{0}(t)$ is updated by

$$
\dot{\hat{\lambda}}_{i}^{0}(t)=-\Gamma_{d_{i}^{0}} z_{2}(t) \operatorname{sgn}\left[z_{2}(t)\right] \chi_{\bar{d} i}(t) .
$$

The controller $\nu_{0}(t)$ is designed as

$$
\begin{aligned}
\nu_{0}(t)= & -\hat{\rho}_{k \nu}(t) \hat{\xi}(t) \varpi(t)+\sum_{i=1}^{l} \chi_{m i}(t)\left(\nu_{d i}(t)\right. \\
& \left.+\hat{\rho}_{i}(t) \zeta_{i}(t)-\hat{\rho}_{i}(t) \eta(t)-\hat{\rho}_{i}(t) r_{2} z_{2}(t)\right),
\end{aligned}
$$

where $\eta(t)$ and $\zeta_{i}(t)$ are defined in (32) and (33) with the estimates $\hat{a}_{i}(t), \hat{b}_{i}(t), \hat{c}_{i}(t), \hat{\vartheta}_{i}(t), \hat{D}_{i}(t)$ being updated by the adaptive laws in (27)-(31); $\hat{\rho}_{i}(t), \hat{\xi}(t)$ and $\hat{\rho}_{k \nu}(t)$ are given by the adaptive laws (37), (41) and (44). $\nu_{d i}(t)$ are designed to compensate unmodeled disturbance $d_{i}(t)$ as

$$
\nu_{d i}(t)=-\frac{1}{\rho_{m}^{0}} \hat{\lambda}_{i}^{0}(t) \operatorname{sgn}\left[z_{2}(t)\right] \chi_{\bar{d} i}(t),
$$

where $\rho_{i}^{0}$ is a known lower bound on $m_{i} k_{\nu}: m_{i} k_{\nu} \geq \rho_{i}^{0}$, and $\hat{\lambda}_{i}^{0}(t)$ is updated by the adaptive law (54).

The above adaptive control scheme leads to

$$
\begin{aligned}
\dot{V}_{2}= & -r_{1} z_{1}^{2}(t)-r_{2} z_{2}^{2}(t) \\
& +\sum_{i=1}^{l}\left(-\frac{m_{i}}{\rho_{m}^{0}} \hat{\lambda}_{i}^{0}(t)\left|z_{2}(t)\right| \chi_{\bar{d} i}(t)+\hat{\lambda}_{i}^{0}(t)\left|z_{2}(t)\right| \chi_{\bar{d} i}(t)\right. \\
& \left.-\left|z_{2}(t)\right| \lambda_{i}^{0} \chi_{\bar{d} i}(t)+z_{2}(t) \bar{d}_{i}(t) \chi_{\bar{d} i}(t)\right) \\
\leq & -r_{1} z_{1}^{2}(t)-r_{2} z_{2}^{2}(t) \leq 0
\end{aligned}
$$


Stability analysis. Similar to the stability analysis in Section $\mathrm{V}$, it is concluded that $z_{1}(t)=x_{1}(t)-x_{m}(t), z_{2}(t)=$ $x_{2}(t)-\alpha_{1}(t), \xi-\hat{\xi}(t), a_{i}-\hat{a}_{i}(t), b_{i}-\hat{b}_{i}(t), c_{i}-\hat{c}_{i}(t)$, $\vartheta_{i}-\hat{\vartheta}_{i}(t), D_{i}-\hat{D}_{i}(t), \lambda_{i}^{0}-\hat{\lambda}_{i}^{0}(t), \rho_{k \nu}-\hat{\rho}_{k \nu}(t), \rho_{i}-\hat{\rho}_{i}(t)$, are bounded, and so are $z_{1}(t), \hat{\xi}(t), \hat{a}_{i}(t), \hat{b}_{i}(t), \hat{c}_{i}(t), \hat{\vartheta}_{i}(t), \hat{D}_{i}(t)$, $\hat{\lambda}_{i}^{0}(t), \hat{\rho}_{k \nu}(t)$ and $\hat{\rho}_{i}(t)$. Then, with the failure compensation controller (48), the boundedness of $\nu_{0}(t)$ is ensured. Further, (52) implies $z_{1}(t) \in L_{2}$ and so $\lim _{t \rightarrow \infty} z_{1}(t)=0$.

For the unknown disturbance bound case, the following conclusion can be obtained:

Theorem 4: The adaptive failure compensation controller (55), with signal (56) and the adaptive scheme (27)-(31), (37), (41), (44), and(54) applied to the system (7)-(8) with actuator failures (13)-(15), guarantees that all closed-loop signals are bounded and the tracking error $e(t)=x_{1}(t)-x_{m}(t)$ satisfies $\lim _{t \rightarrow \infty} e(t)=0$.

The failure compensation controller designed in this section is for the case when the bounds on disturbances are unknown. To deal with the unknown bounds, the adaptive law (54) is utilized to estimate the unknown designed parameter $\lambda_{i}^{0}$ (shown in (36) or (43) or (50)), which is designed for the known bound case. The proposed method can be easily extended to the unparameterizable failure case, through an additional adaptive law to estimate the unknown designed parameter $\delta_{i}^{0}$. To prevent possible parameter drift in the adaptive law (54) and other adaptive laws in the presence of possible system noises and additional disturbances, robust adaptation techniques in [27] can be employed.

\section{FAILURE COMPENSATION CONTROLLER FOR UnParameterizable System Parameters}

In the above sections, the system parameters of the highspeed train dynamic model are completely parameterized, and the bounded disturbance is used to express the unparameterizable uncertain time variations. In this section, we show that even when there are unparameterizable system parameters with unknown upper bounds, we can design the failure compensation controller to ensure boundedness of the tracking errors of the closed-loop system.

The longitudinal motion dynamics (4) with unparameterizable system parameters can be expressed as:

$$
\begin{aligned}
\dot{x}_{1}(t)= & x_{2}(t), \\
\dot{x}_{2}(t)= & m(t) F(t)-a(t)-(b(t)+\Delta b(t)) x_{2}(t) \\
& -(c(t)+\Delta c(t)) x_{2}^{2}(t)-g \vartheta(t)-0.004 D(t) \\
& +\bar{d}(t),
\end{aligned}
$$

where $m(t), a(t), b(t), c(t), \vartheta(t), D(t)$, and $\bar{d}(t)$ are defined in (9) and (10); $\Delta b(t)$ and $\Delta c(t)$ are bounded unparameterizable system parameters, and the upper bounds on $|\Delta b(t)|$ and $|\Delta c(t)|$ could be unknown. The unparameterizable part of parameter $a(t)$ can belong to $\bar{d}(t)$.

Recall the actuator failure (13)-(15). The control signal $\nu_{0}(t)$ is designed as

$$
\begin{aligned}
\nu_{0}(t)= & -\hat{\rho}_{k \nu}(t) \hat{\xi}^{T}(t) \varpi(t)+\sum_{i=1}^{l} \chi_{m i}(t)\left(\nu_{d i}(t)+\hat{\rho}_{i}(t) \nu_{\Delta}(t)\right. \\
& \left.+\hat{\rho}_{i}(t) \zeta_{i}(t)-\hat{\rho}_{i}(t) \eta(t)-\hat{\rho}_{i}(t) r_{2} z_{2}(t)\right)
\end{aligned}
$$

where $\nu_{\Delta}(t)$ is designed to compensate unknown $\Delta b(t)$ and $\Delta c(t)$ for stability; $\eta(t)$ and $\zeta_{i}(t)$ are defined in (32) and (33) with the estimates $\hat{a}_{i}(t), \hat{b}_{i}(t), \hat{c}_{i}(t), \hat{\vartheta}_{i}(t), \hat{D}_{i}(t)$ being updated by the adaptive laws in (27)-(31); $\hat{\xi}(t)$ and $\hat{\rho}_{k \nu}(t)$ are given by the adaptive laws (41) and (44); $\nu_{d i}(t)$ is given as (43). $\hat{\rho}_{i}(t)$ is updated from the adaptive law

$$
\begin{aligned}
\dot{\hat{\rho}}_{i}(t)= & -\gamma_{m i} z_{2}(t)\left(-\nu_{\Delta}(t)+\zeta_{i}(t)-\eta(t)\right. \\
& \left.-r_{2} z_{2}(t)\right) \chi_{m i}(t) .
\end{aligned}
$$

The design signals $\nu_{\Delta}(t)$ is given by

$$
\nu_{\Delta}(t)=-k_{1} z_{2}(t) x_{2}^{2}(t)-k_{2} z_{2}(t) x_{2}^{4}(t),
$$

with $k_{1}>0$ and $k_{2}>0$.

Using the Lyapunov function (40), the above adaptive control scheme leads to

$$
\begin{aligned}
\dot{V}_{2}= & -r_{1} z_{1}^{2}(t)-r_{2} z_{2}^{2}(t)+\sum_{i=1}^{l}\left(-\frac{m_{i} k_{\nu}}{\rho_{i}^{0}} \lambda_{i}^{0}\left|z_{2}(t)\right| \chi_{\bar{d} i}(t)\right. \\
& \left.+z_{2}(t) \bar{d}_{i}(t) \chi_{\bar{d} i}(t)\right)+\nu_{\Delta}(t)-\Delta b(t) z_{2}^{2}(t) x_{2}(t) \\
& -\Delta c(t) z_{2}^{2}(t) x_{2}^{2}(t) .
\end{aligned}
$$

Stability analysis. Taking $\nu_{\Delta}(t)$, we obtain

$$
\begin{aligned}
\dot{V}_{2} \leq & -r_{1} z_{1}^{2}(t)-r_{2} z_{2}^{2}(t)+\left(-k_{1} z_{2}^{2}(t) x_{2}^{2}(t)\right. \\
& \left.+b_{0}\left|z_{2}(t)\right|\left|x_{2}(t)\right|-k_{2} z_{2}^{2}(t) x_{2}^{4}(t)+c_{0}\left|z_{2}(t)\right| x_{2}^{2}(t)\right) .
\end{aligned}
$$

in which $b_{0}$ and $c_{0}$ are (unknown) upper bounds on $|\Delta b(t)|$ and $|\Delta c(t)|$. The term

$$
\begin{aligned}
& -k_{1} z_{2}^{2}(t) x_{2}^{2}(t)+b_{0}\left|z_{2}(t)\right|\left|x_{2}(t)\right|-k_{2} z_{2}^{2}(t) x_{2}^{4}(t) \\
& \quad+c_{0}\left|z_{2}(t)\right| x_{2}^{2}(t)
\end{aligned}
$$

attains a maximum value $\left(\frac{b_{0}^{2}}{4 k_{1}}+\frac{c_{0}^{2}}{4 k_{2}}\right)$ at $\left|x_{2}(t)\right|=\frac{k_{1} c_{0}}{k_{2} b_{0}}$ and $\left|z_{2}(t)\right|=\frac{k_{2} b_{0}^{2}}{2 k_{1}^{2} c_{0}}$. Therefore,

$$
\dot{V}_{2} \leq-r_{1} z_{1}^{2}(t)-r_{2} z_{2}^{2}(t)+\left(\frac{b_{0}^{2}}{4 k_{1}}+\frac{c_{0}^{2}}{4 k_{2}}\right) .
$$

Since $r_{1}$ and $r_{2}$ are positive constants, it is always true that $\dot{V}_{2}$ is negative outside some ball. It concludes that for any initial states $z_{1}(0)$ and $z_{2}(0)$, the solution of the closed-loop system is uniformly bounded. The term $\nu_{\Delta}(t)(62)$ is called nonlinear damping. Recall the definition of $z_{1}(t)$ and $z_{2}(t)$ in (18) and (19). It can concluded that the distance and speed tracking errors are bounded. We give the conclusion in the following theorem.

Theorem 5: The adaptive failure compensation controller (60), with the adaptive scheme (27)-(33), (41), (43) and (44) 
applied to the system (58)-(59) with actuator failures (13)-(15), guarantees that all the closed-loop signals are bounded.

Remark 4: For the nonlinear damping term $\nu_{\Delta}(t)$, there are two designed constants $k_{1}>0$ and $k_{2}>0$, which determine the convergent ball of $\dot{V}_{2}$. From (65), it can be seen that $k_{1}$ and $k_{2}$ can be chosen to make the constant term $\frac{b_{0}^{2}}{4 k_{1}}+\frac{c_{0}^{2}}{4 k_{2}}$ as small as desired, i.e., the tracking errors can achieve an arbitrary degree of accuracy. The larger the constants $k_{1}>0$ and $k_{2}>0$ are, the smaller the tracking errors $z_{1}$ and $z_{2}$ are. In practice, $k_{1}$ and $k_{2}$ are chosen to satisfy the acceptable accuracy of the tracking errors.

For the high-speed train dynamic model (58)-(59), the timevarying indicator functions are used to parameterize the timevarying system parameter for adaption, while the remaining unparameterizable parameters are kept, which are dealt with using nonlinear damping $\nu_{\Delta}(t)$ in control, to an arbitrary degree of accuracy.

From now on, several different cases about the system failures and disturbances with unknown parameters have been investigated to design the adaptive controllers. Considering the repeatable pattern of the high-speed trains, the case that the bounds of the disturbances and unparameterizable failures are known is the most popular. Thus, the adaptive controllers proposed in this paper satisfy the real requirements of the high-speed trains.

\section{Simulation STUdY}

In this section, the simulation study on a high-speed train are presented for various operation conditions, to demonstrate the effectiveness of the proposed adaptive controllers. The parameters in the simulation are from a real CRH type train, which contains 8 vehicles (4 locomotives and 4 carriages).

Simulation conditions. According to [1], there are several operating conditions including acceleration, reacceleration, constant speed, deceleration, redeceleration, and slowing down until fully stop. The tracking distance curve, as shown in Fig. 1 (solid line), is considered.

Form the tracking curve in Fig. 1, the train does not stop. So the mass of the train is chosen as a constant, i.e., $M_{i}=M=$ 400 ton. Considering the tunnel, slope and curvature during the train running, 4 modes are set for the healthy system, in which the parameters $a_{i}(\mathrm{kN}), b_{i}(\mathrm{kN} \mathrm{s} / \mathrm{m}), c_{i}\left(\mathrm{kN} \mathrm{s}^{2} / \mathrm{m}^{2}\right)$, $\vartheta_{i}$ (degree), and $D_{i}$ are defined in equations (9)-(10), and the unit of time is sec.

(i) During $0 \leq t<400$, the train starts. Then, $a_{1}=8.63 \times$ $10^{-3}, b_{1}=7.295 \times 10^{-6}, c_{1}=1.12 \times 10^{-6}, \theta_{1}=0$, and $D_{1}=0$.

(ii) For $400 \leq t<800$, the train enters a tunnel. Then, $a_{2}=8.63 \times 10^{-3}, b_{2}=7.295 \times 10^{-6}, c_{2}=9.12 \times 10^{-6}$, $\theta_{2}=0$, and $D_{2}=0$.

(iii) At $t=800$, the train leaves the tunnel and travels in a curvature and slope track. During $800 \leq t<1400$, $a_{3}=8.63 \times 10^{-3}, b_{3}=7.295 \times 10^{-6}, c_{3}=1.12 \times 10^{-6}$, $\theta_{3}=0.018$, and $D_{3}=0.34$.

(iv) After $t=1400$, the train moves in the open air and horizontal track to slow down until fully stop. For

$$
\begin{aligned}
& 1400 \leq t<2000, a_{4}=8.63 \times 10^{-3}, b_{4}=7.295 \times 10^{-6}, \\
& c_{4}=1.12 \times 10^{-6}, \theta_{4}=0, \text { and } D_{4}=0 .
\end{aligned}
$$

The indicator functions for the parameters $a, b, c, D$ are chosen as 0 - 1 functions, while $\chi_{\theta 3}=0.25(t-800)$ for $t \in[800,804]$.

Case 1 (healthy mode). For the healthy high-speed train, the disturbances are set as:

$$
d(t)= \begin{cases}100 \sin (0.03 t), & t \in[0,400) ; \\ 200\left(1-e^{-10 t}\right), & t \in[400,800) ; \\ 100, & t \in[800,1400) \\ 0, & t \in[1400,2000]\end{cases}
$$

Choose the initial sates as $x(0)=\left[\begin{array}{ll}0.55 & 0\end{array}\right]^{T}$, and the initial parameter estimates as $90 \%$ of their nominal values. The gains of the adaptive laws in (27)-(31) are chosen as 2 .

Case 2 (parameterizable failure mode). In this case, 16 motors are considered to provide the traction force, i.e., $n=$ 16. Consider the same control scheme is applied for each same type motor. The effectiveness of the failed motors is equivalent to the failure in one motor. Here, one motor failure is taken into consideration, which is a constant failure at the beginning and then becomes a time-varying failure. At last, the failed motor stops completely. The failure is expressed as: $F_{\alpha}$ fails for some $\alpha \in\{1, \ldots, 16\}$,

$$
\begin{aligned}
& F_{\alpha} \\
& = \begin{cases}2 \times 10^{5}, & t \in[600,1000) ; \\
2 \times 10^{5}(1+\sin (0.05 t-30)), & t \in[1000,1200) ;(67) \\
0, & t \in[1200,2000] .\end{cases}
\end{aligned}
$$$$
F_{\beta}=\nu_{\beta}, \beta \neq \alpha, \beta \in\{1, \ldots, 16\} \text {. }
$$

The initial conditions are chosen as $x(0)=\left[\begin{array}{lll}-0.55 & 0\end{array}\right]^{T}$, and the initial parameter estimates are $80 \%$ of their ideal values.

Case 3 (unparameterizable failure mode). The parameterizable failure part is same as $F_{\alpha}$ (67) in case 2, while the unparameterizable failure term $\bar{\delta}_{j}(t)$ is chosen as $\bar{\delta}_{j}(t)=3 \times$ $10^{4} \sin (0.07 t)$, which is assumed to be the unparameterizable part and is not considered as the basis signal. It can be easily obtained the bound of the unparameterizable part of the failure, i.e., $3 \times 10^{4}$. The initial conditions are chosen as $x(0)=\left[\begin{array}{ll}0 & 0\end{array}\right]^{T}$, and the initial parameter estimates are $90 \%$ of their ideal values.

Case 4 (unknown disturbance mode). In this case, the failure is expressed as that in Case 2, and the disturbances are set as:

$$
\begin{aligned}
& d(t) \\
& = \begin{cases}100 \sin (0.03 t)+500 \sin (0.09 t), & t \in[0,400) ; \\
200\left(1-e^{-10 t}\right)+500 \sin (0.09 t), & t \in[400,800) ; \\
100+500 \sin (0.09 t), & t \in[800,1200) ; \\
0, & t \in[1200,2000] .\end{cases}
\end{aligned}
$$

The bounds on the disturbances are unknown for the simulation. The initial conditions are chosen as $x(0)=\left[\begin{array}{ll}-0.65 & 0\end{array}\right]^{T}$, and the initial parameter estimates are $90 \%$ of their ideal values.

Case 5 (unparameterizable system parameters). In this case, the disturbance $d(t)$ and failure are expressed as (66) and (67), respectively. The unparameterizable system parameters $\Delta b(t)$ and $\Delta c(t)$ are set as: $\Delta b(t)=0.001 \sin (0.02 t)$, $\Delta c(t)=0.001 \sin (0.02 t)$. The initial conditions are chosen as 
$x(0)=\left[\begin{array}{ll}-0.45 & 0\end{array}\right]^{T}$, and the initial parameter estimates are $90 \%$ of their ideal values.

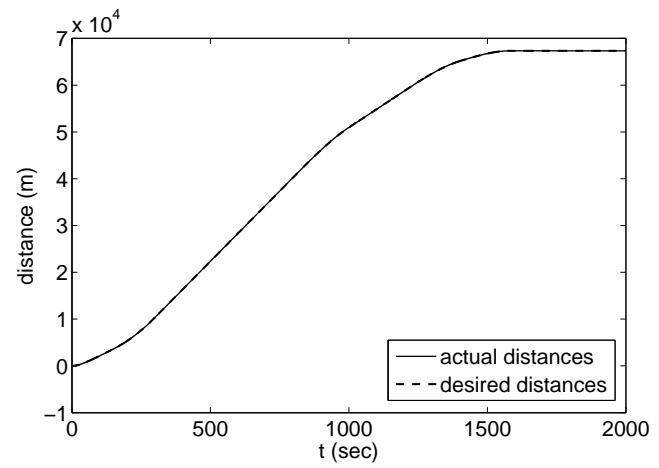

(a) distance trajectories

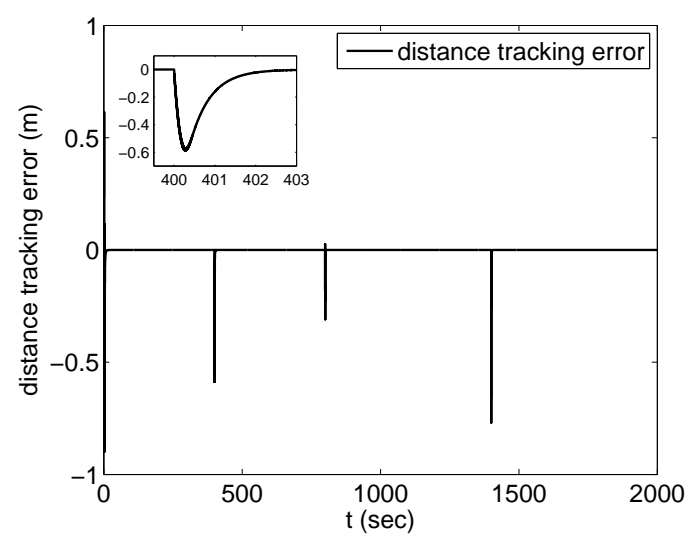

(b) tracking error

Fig. 1: Distance trajectory tracking performance for the healthy model.

Simulation results. Fig. 1 shows the simulation results of the healthy system including the desired distance (solid) and plant distance (dashed). Fig. 2 shows the distance tracking errors for cases 1-5. From Fig. 2, it can be seen that there exist some transit responses, which are caused by the changes of the system parameters or the occurrences of failures. The simulation results show that the proposed adaptive controller can achieve the close-loop stability and asymptotic tracking properties of the train even in the presence of parameters changes and actuator failures.

\section{COnClusions}

The adaptive failure compensation problem for high-speed trains with time-varying parameters, disturbances and actuator failures, is studied. The time-varying indicator function is introduced to parameterize the time-varying system parameters. The adaptive failure compensation controllers with design procedures, both for healthy system, and faulty system with disturbances, have been developed. Simulation results further confirm the desired performance of the proposed scheme.

\section{ACKNOWLEDGMENT}

Zehui Mao carried out this work, when she was a visiting scholar at the Department of Electrical and Computer Engi-

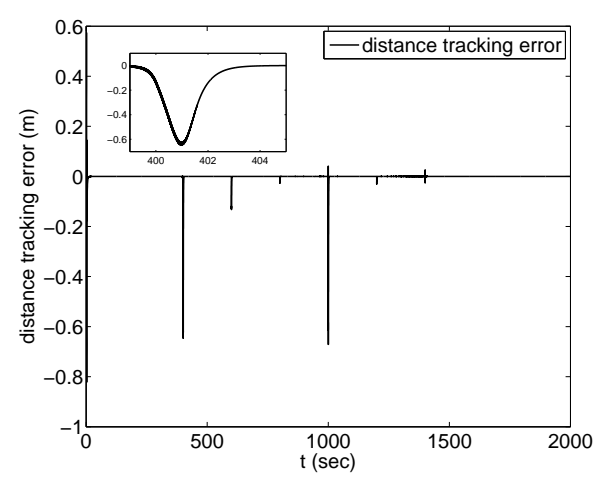

(a) faulty model with known disturbance bounds

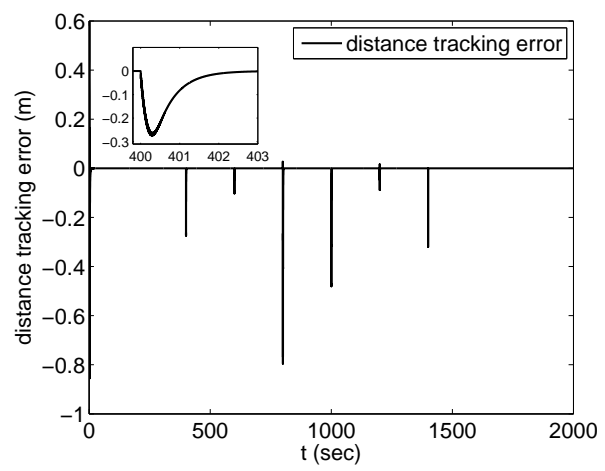

(b) unparameterizable faulty model

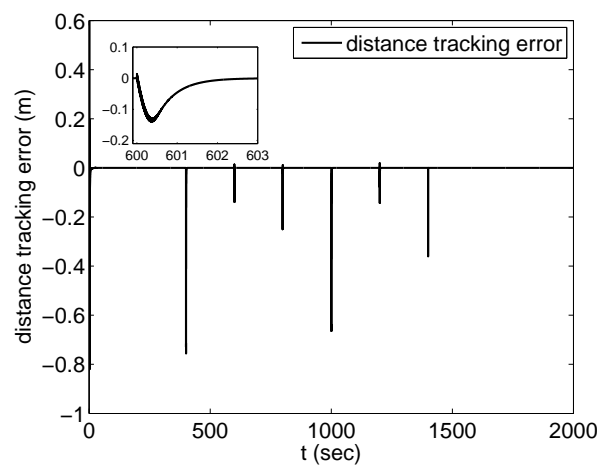

(c) faulty model with unknown disturbance bounds

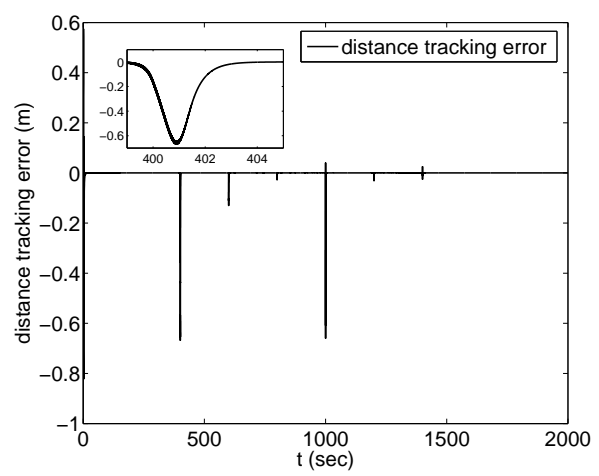

(d) faulty model with unparameterizable parameters

Fig. 2: Distance tracking errors. 
neering, University of Virginia, USA. The author gratefully acknowledges the support that she has received from the Department of Electrical and Computer Engineering, University of Virginia, USA.

\section{REFERENCES}

[1] Y. D. Song, W. T. Song, A novel dual speed-curve optimization based approach for energy-saving operation of high-speed trains, IEEE Trans. Intell. Transp. Syst., vol. 17, no. 6, pp. 1564-1575, 2016.

[2] J. Eberhard, Railway infrastructure and the development of high-speed rail in Germany, Railw. Tech. Rev., vol. 2, pp. 3-11, 2005.

[3] Y. Chen, H. R. Dong, J. h. Lv, X, B. Sun, L, Guo, A super-twistinglike algorithm and its application to train operation control with optimal utilization of adhesion force, IEEE Trans. Intell. Transp. Syst., vol. 17, no. 11, pp. 3035-3044, 2016

[4] R. R. Wang, J. M. Wang, Passive actuator fault-tolerant control for a class of overactuated nonlinear systems and applications to electric vehicles, IEEE Trans. Veh. Technol., vol. 62, no. 3, pp. 972-985, 2013.

[5] X. He, Z. D. Wang, L. Qin, D. H. Zhou, Active fault tolerant control for an Internet-based networked three-tank system, IEEE Trans. Control Syst. Technol., vol. 24, no. 6, pp. 2150-2157, 2016.

[6] X. He, Z. D. Wang, Y. Liu, D. H. Zhou, Least-squares fault detection and diagnosis for networked sensing systems using direct state estimation approach, IEEE Trans. Ind. Informatics, vol. 9, no. 3, pp. 1670-1679, 2013.

[7] B. Jiang, Z. F. Gao, P. Shi, Y. F. Xu, Adaptive fault-tolerant tracking control of near-space vehicle using Takagi-Sugeno fuzzy models, IEEE Trans. on Fuzzy Syst., vol. 18, no. 5, pp. 1000-1007, 2010.

[8] Y. Wang, Y. D. Song, H. Gao, F. L. Lewis, Distributed fault-tolerant control of virtually and physically interconnected systems with application to high-speed trains under traction/braking failures, IEEE Trans. Intell. Transp. Syst., vol. 17, no. 2, pp. 535-545, Feb. 2016.

[9] Z. H. Mao, G. Tao, B. Jiang, X. Yan, Adaptive compensation of traction system actuator failures for high-speed trains, IEEE Trans. Intell. Transp. Syst., vol. 18, no. 11, pp. 2950-2963, 2017.

[10] Y. D. Song, Q. Song, W. C. Cai, Fault-tolerant adaptive control of highspeed trains under traction/braking failures: a virtual parameter-based approach, IEEE Trans. Intell. Transp. Syst., vol. 15, no. 2, pp. 737-748, Apr. 2014.

[11] H. H. Ji, Z. Hou, R. Zhang, Adaptive iterative learning control for high-speed trains with unknown speed delays and input saturations, IEEE Trans. Autom. Sci. Eng., vol.13, no. 1, pp. 260-273, Jan. 2016.

[12] Z. Ma, J. Xie, H. Li, Q. Sun, Z. Si, J. Zhang, J. Guo, The role of data analysis in the development of intelligent energy networks, IEEE Network, Vol. 31, no. 5, pp. 88-95, 2017.

[13] Z. Ma, J. H. Xue, A. Leijon, Z.H. Tan, Z. Yang, J. Guo, Decorrelation of neutral vector variables: theory and applications, IEEE Trans. Neural Networks and Learning Systems, http://doi.org/10.1109/TNNLS.2016.2616445, 2017.

[14] Z. Ma, A. E. Teschendorff, A. Leijon, Y. Qiao, H. Zhang, J. Guo, Variational bayesian matrix factorization for bounded support data, IEEE Trans. Pattern Analysis and Machine Intelligence, Vol. 37, no. 4, pp. 876-889, 2015.

[15] Y. Chen, J. M. Wang, Adaptive vehicle speed control with input injections for longitudinal motion independent road frictional condition estimation, IEEE Trans. Veh. Technol., vol. 60, no. 3, pp. 839-848, 2011.

[16] G. Tao, S. M. Joshi, X. L. Ma, Adaptive state feedback and tracking control of systems with actuator failures, IEEE Trans. Autom. Control, vol. 46, no.1, pp. 78-95, 2001.

[17] G. Tao, S. H. Chen, S. M. Joshi, An adaptive actuator failure compensation controller using output feedback, IEEE Trans. Autom. Control, vol. 47, no. 3, pp. 506-511, 2002.

[18] B. P. Rochard, F. Schmid, A review of methods to measure and calculate train resistances, Proceedings of the Institution of Mechanical Engineers, Part F: Journal of Rail and Rapid Transit, vol. 214, no. 4, pp. 185-199, 2000.

[19] V. K. Garg, Dynamics of railway vehicle systems, Academic Press, 1984.

[20] W. C. Cai, D. Y. Li, B. Liu, Y. Song, Uniform rolling-wear-based robust adaptive control of high-speed trains in the presence of actuator differences, IEEE Trans. Intell. Transp. Syst., vol. 17, no. 12, pp. 35913601, Dec., 2016.
[21] H. S. Hwang, Control strategy for optimal compromise between trip time and energy consumption in a high-speed railway, IEEE Trans. Syst. Man Cybern. -Part A: Syst. and Humans, vol. 28, no. 6, pp. 791802, Nov. 1998.

[22] M. Faieghi, A. Jalali, and S. K. M. Mashhadi, Robust adaptive cruise control of high speed trains, ISA Trans., vol. 53, no. 2, pp. 533-541, Mar. 2014.

[23] X. M. Yao, L. Guo, Composite anti-disturbance control for Markovian jump nonlinear systems via disturbance observer, Automatica, vol. 49, no. 8, pp. 2538-2545, 2013.

[24] X. M. Yao, L. Guo, L. G. Wu, H. R. Dong, Static anti-windup design for nonlinear Markovian jump systems with multiple disturbances, Information Sciences, vol. 418, pp. 169-183, 2017.

[25] A. F. Filippov, Differential equations with discontinuous right hand sides, Amer. Math. Soc. Trans., vol. 42, pp. 199-231, 1964.

[26] V. I. Utkin, Sliding Modes in Control Optimization, Berlin, Germany: Springer-Verlag, 1991.

[27] P. A. Ioannou, J. Sun, Robust Adaptive Control, Prentice-Hall, Englewood Cliffs, NJ, 1996.

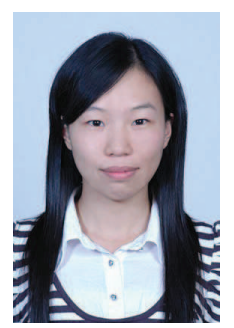

Zehui Mao received her Ph.D. degree in Control Theory and Control Engineering from Nanjing University of Aeronautics and Astronautics, Nanjing, China, in 2009. She is now an associate professor at the College of Automation Engineering in Nanjing University of Aeronautics and Astronautics, China. And she was a visiting scholar in University of Virginia. She worked in the areas of fault diagnosis, with particular interests in nonlinear control systems, sampled-data systems and networked control systems. Her current research interests include fault diagnosis and fault-tolerant control of systems with disturbance and incipient faults, and high speed train and spacecraft flight control applications.

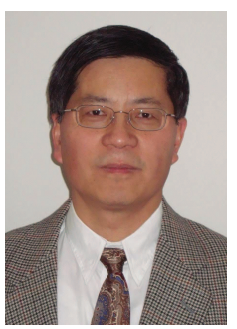

Gang Tao (S'84-M'89-SM'96-F'07) received the B.S. degree from the University of Science and Technology of China, Hefei, China, in 1982, and the M.S. and Ph.D. degrees from the University of Southern California, Los Angeles, CA, USA, during 1984-1989. He is currently a Professor at the University of Virginia, Charlottesville, VA, USA. He worked in the areas of adaptive control, with particular interests in adaptive control of system$\mathrm{s}$ with multiple inputs and multiple outputs and with nonsmooth nonlinearities and actuator failures, in stability and robustness of adaptive control systems, and in passivity characterizations of control systems. He has authored or coauthored six books, and more than 400 technical papers and book chapters. His current research interests include adaptive control of systems with uncertain actuator failures and nonlinearities, with structural damage and sensor uncertainties and failures, adaptive approximation-based control, and resilient aircraft and spacecraft flight control applications. He has served as an Associate Editor of Automatica, the International Journal of Adaptive Control and Signal Processing, and the IEEE TRANSACTIONS ON AUTOMATIC CONTROL, a Guest Editor of the Journal of Systems Engineering and Electronics, and an Editorial Board Member of the International Journal of Control, Automation and Systems. 


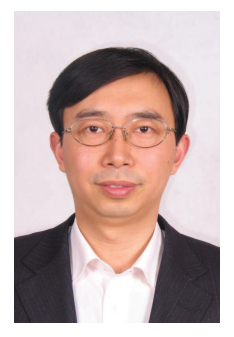

Bin Jiang the Ph.D. degree in Automatic Control from Northeastern University, Shenyang, China, in 1995. He had ever been postdoctoral fellow, research fellow, invited professor and visiting professor in Singapore, France, USA and Canada, respectively. Now he is a Chair Professor of Cheung Kong Scholar Program in Ministry of Education and Dean of College of Automation Engineering in Nanjing University of Aeronautics and Astronautics, China. He currently serves as Associate Editor or Editorial Board Member for a number of journals such as IEEE Trans. On Control Systems Technology; Int. J. Of $\mathrm{C} \equiv$ Automation and Systems; Journal of Astronautics; Control and Decision, He is a senior member of IEEE, Chair of Control Systems Chapter in IEEE Nanjing Section, a member of IFAC Technical Committee on Fault Detection, Supervision, and Safety of Technical Processes. His research interests include intelligent fault diagnosis and fault tolerant control and their applications to helicopters, satellites and high-speed trains.

$\mathrm{He}$ has been the principle investigator on several projects of National Natural Science Foundation of China. He is the author of 8 books and over 200 referred international journal papers and conference papers. He won First Class Prize of Natural Science Award of Ministry of Education of China in 2015.

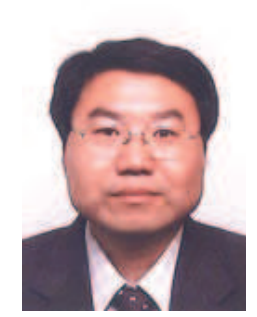

Xing-Gang Yan received B.Sc. degree from Shanxi Normal University, in 1985, M.Sc. degree from Qufu Normal University in 1991, and Ph.D. degree in Engineering from Northeastern University, China in 1997. Currently, he is a Senior Lecturer at the University of Kent, UK. He was a Lecturer at Qingdao University, China from 1991 to 1994. He worked as a Research Fellow/Associate in the University of Hong Kong, China, Nanyang Technological University, Singapore and the University of Leicester, UK. He is the Editor-in-Chief of the International Journal of Engineering Research and Science and Technology. His research interests include sliding mode control, decentralised control, fault detection and isolation, control and observation of nonlinear systems and time delay systems with applications. 